\title{
Polarization dependence of four-wave mixing in a degenerate two-level system.
}

\author{
A. Lezama ${ }^{1,2}$, G.C. Cardoso ${ }^{1}$, and J.W.R. Tabosa ${ }^{1}$ \\ 1 Departamento de Fí sica, Universidade Federal de Pernambuco, \\ 50670-901 Recife, PE - Brazil \\ ${ }^{2}$ Instituto de Física, Facultad de Ingeniería, C. Postal.30. \\ 11000, Montevideo, Uruguay \\ (May 2, 2019)
}

Nearly degenerate four-wave mixing (NDFWM) within a closed degenerate two-level atomic transition is theoretically and experimentally examined. Using the model presented by A. Lezama et al [Phys. Rev. A 61, 013801 (2000)] the NDFWM spectra corresponding to different pump and probe polarization cases are calculated and discussed. The calculated spectra are compared to the observation of NDFWM within the $6 S_{1 / 2}(F=4) \rightarrow 6 P_{3 / 2}(F=5)$ transition of cesium in a phase conjugation experiment using magneto optically cooled atoms.

\section{INTRODUCTION.}

The generation of new optical fields is the most spectacular consequence of the intrinsic non-linearity of the light-matter interaction. Four-wave mixing (FWM), i.e. the generation of a fourth field as the result of the interaction of a material sample with three electromagnetic fields is the simplest non-linear optics generation process allowed in every material [1.2].

This paper is concerned with nearly degenerate four wave mixing (NDFWM) occurring when two of the three incident fields (designated as pump fields) have the same frequency $\omega_{1}$ and the third field (probe field) has an independent frequency $\omega_{2} \equiv \omega_{1}+\delta$ which is tunable around $\omega_{1}$. Of particular interest is the case where all fields are nearly resonant with the same optical atomic transition. In such case the spectroscopic study of the NDFWM signal as a function of the probe to pump frequency offset can provide useful information about the atomic dynamics. NDFWM was used by Rothberg and Bloembergen [3] for studying collisional dynamics in sodium vapor. Boyd et al [4] have studied theoretically the atomic polarization and the propagation of the probe and NDFWM waves in the case of a pure two-level system (PTLS) driven by a arbitrarily intense pump. Their work illustrate the critical influence of the atomic damping mechanism in the FWM process. Steel et al [5] explored NDFWM in both open and closed PTLS. Berman and coworkers [6] clearly established the suitability of NDFWM for the determination of all relevant relaxation parameters. Andersen and coworkers analyzed the NDFWM process in PTLS in terms of the dressed state picture [7]. Their work stresses the importance of the initial atom-field state on the FWM yield.

In spite of the fact that the experiments on NDFWM generally involve degenerate energy levels, most experiments to date were carried under conditions where the level degeneracy does not play an essential role. In such cases, simplified theoretical models using two-level schemes were successfully used. Nevertheless, the role of the level degeneracy for NDFWM was clearly appreciated by Berman and coworkers [6] who stressed the importance of the non-conservation in the evolution of the multipolar expansion terms of the atomic density matrix, for the observation of sub-natural resonances in the NDFWM signal spectrum. Their work include a detailed calculation, valid for degenerate atomic levels, of the nonlinear atomic polarization to the lowest order in the incident fields. Recently, a model allowing the numerical investigation of the complete response of a degenerate two-level system (DTLS) to first order in the probe field for an arbitrarily intense pump and arbitrary pump and probe polarizations [8] was presented.

Recent experimental observations illustrate the need for a deeper understanding of the role of the level degeneracy in the non linear response of DTLS. An important example is provided by the spectroscopic response of alkaline atoms in a magneto-optical trap (MOT). In this system the atoms are submitted to the excitation of the trapping beams which are quasi resonant with a closed degenerate transition. The absorption [9, 10] and FWM 11, 12 spectra of magneto-optically trapped atoms present characteristic features resulting from the level degeneracy. Recently, the use of DTLS for efficient and highly selective NDFWM generation in an atomic vapor was reported 13.

The aim of this paper is to address the role of the level degeneracy in NDFWM on a closed transition between two degenerate atomic levels. The dependence of NDFWM generation on the intensity of the pumping field and the polarizations of the pump and probe will be analyzed. In the next section, NDFWM spectra calculated for an homogeneous ensemble of DTLS under different conditions are presented. The following section is devoted to the experimental observation of NDFWM in cold cesium atoms. Discussion of the experimental results in regard to the theoretical predictions follows. 


\section{THEORETICAL PREDICTIONS.}

The NDFWM generation in an homogeneous ensemble of DTLS is analyzed using the semiclassical procedure already presented in [8]. In Fig. 1 the essential elements of this model are reminded. The two-level atoms are driven by a pump field of frequency $\omega_{1}$ of arbitrary intensity and polarization. The reduced pump field Rabi frequency is $\Omega_{1}$. The driven atoms are probed by a weak field of frequency $\omega_{2} \equiv \omega_{1}+\delta$ and arbitrary polarization. Both fields are nearly resonant with the atomic transition (transition frequency $\hbar \omega_{A}$ ) between the ground level $g$ of total angular momentum $F_{g}$ and the excited level $e$ of total angular momentum $F_{e}$. Having in mind the experimental observation presented below, we will restrict our attention to closed atomic transitions with $F_{e}=F_{g}+1$. Level $e$ decays back into $g$ through spontaneous emission at rate $\Gamma$. In addition to this relaxation mechanism a state independent decay rate $\gamma(\gamma \ll \Gamma)$ is assumed which represents the departure of the atoms from the interaction zone. This departure is compensated at steady state by the arrival (pumping term $\gamma \rho_{0}$ ) of "fresh" atoms isotropically distributed in the ground level. ( $\rho_{0}$ is the system density matrix in the absence of any applied field). Since no other relaxation mechanism exist for the ground state, $\gamma$ effectively plays the role of a ground state relaxation rate. The calculation is exact to all orders in the pump field and to first order in the probe field. The spectra represent the variation of the squared modulus of the atomic polarization induced at the frequency $\omega_{3} \equiv 2 \omega_{1}-\omega_{2} \equiv \omega_{1}-\delta$ as a function of the probe to pump frequency offset $\delta$. Since the calculation corresponds to the response of an homogeneous ensemble of atoms at rest, no propagation effects such as phase matching, spatial emission pattern of the generated wave or beam absorption (or amplification) are accounted for.

Before presenting the spectra calculated for DTLS, let us remind the main features of the NDFWM spectra for PTLS [4]5]. Figure 2 represent the spectra of the NDFWM power as a function of $\delta$ for $\Delta=0$ and $\Delta=2 \Gamma$ $\left(\Delta \equiv \omega_{A}-\omega_{1}\right)$ for different values of the pump field Rabi frequency $\Omega_{0}$. We restrict ourselves to closed transition where spontaneous emission is the only relaxation mechanism. For all values of $\Omega_{0}$, the spectra present two symmetric sidebands at $\delta= \pm \Omega$ where $\Omega \equiv \sqrt{\Omega_{0}^{2}+\Delta^{2}}$ is the generalized Rabi frequency. The width of these two peaks is determined by $\Gamma$. When $\Omega_{0} \gtrsim \Delta, \Gamma$ a third peak develops at $\delta=0$ whose width is also determined by $\Gamma$. Only when $\Omega \sim \Gamma$ the central peak overcomes the two sidebands. As discussed in [4,5], the relative amplitude of peak at $\delta=0$ is determined by the coherence relaxation rate. In the present case this rate equals $\Gamma / 2$ since only radiative decay is assumed. When the transition is open and when the escaping rates out of the two-level systems are different for the upper and lower level, an additional peak appears at $\delta=0$ with a width determined by the lower level relaxation rate [5]. Finally let us mention that in PTLS the maximum NDFWM power is obtained for $\Omega_{0} \sim \Gamma$.

We examine now the NDFWM power spectra for a DTLS with $F_{g}=1$ and $F_{e}=2$ (no magnetic field present). Three polarization cases were considered: circular and equal pump and probe polarizations, parallel linear pump and probe polarizations and linear and perpendicular pump and probe polarizations. The corresponding level schemes are shown in Fig. 3 where an appropriate choice of the quantization axis is made in each case.

Figure 4 shows the spectra calculated for pump and probe fields with the same circular polarization $\left(\sigma^{+}\right)$. As expected, due to the optical pumping of the population towards the Zeeman sublevel in $g$ and $e$ with the highest magnetic quantum number (see Fig. 3 a), the system behaves as a PTLS in the limit of large $\Omega_{1}$. However, striking differences appear for $\Omega_{1} \lesssim \Gamma$ in which case the Zeeman optical pumping is only partial. Notice the presence at $\delta=0$ of narrow resonances: a peak for $\Omega_{1}=\Gamma$, $\Delta=2 \Gamma$ and a $\operatorname{dip}$ for $\Omega_{1}=\Gamma, \Delta=0$ and $\Omega_{1}=2 \Gamma$, $\Delta=2 \Gamma$. The width of this narrow resonance is determined by $\gamma$. In this configuration, the pump and probe fields interact with pairs of Zeeman sublevels of $e$ and $g$ with $m_{e}=m_{g}+1$ ( $m_{i}$ is a magnetic quantum number). Coupling between different pairs occurs only via spontaneous emission. NDFWM signal arises from the coherent contribution of different Zeeman sublevels pairs excited by the field. Due to spontaneous emission, a given pair is not a close system in which case, a narrow structure (width $\gamma$ ) is expected at $\delta=0$ [5]. As observed in Fig. 4 the sign of this narrow feature may vary depending on the pump field intensity and detuning. It is the result of the quantum interference of the contributions from the different Zeeman sublevels pairs to the non linear atomic polarization. A similar situation occurs in the case of parallel linear polarizations discussed next.

Figure 5 refers to the spectra calculated for pump and probe fields with parallel linear polarizations. Unlike the previous case, in the limit of large $\Omega_{1}$, the system does not behave as a PTLS. Instead, taking as the quantization axis the direction of the common pump and probe polarization, it should be seen as three $\left(2 F_{g}+1\right)$ two-level systems linked through spontaneous emission (Fig. 3 b). Each two-level system consist of a two Zeeman sublevels with $m_{g}=m_{e}$. Since the strength of the atom field coupling dependents only on $\left|m_{g}\right|$, two different values of the pump Rabi frequency occur in the present example. This explains the splitting observed in the two sidebands for large $\Omega_{1}$. At low pump intensities a narrow resonance (width determined by $\gamma$ ) appears at $\delta=0$ for $\Delta=2 \Gamma$. Also a narrow dip is present at $\delta=0$ for $\Delta=0$ and $\Omega_{1}<\Gamma$ (not shown in the figure). The appearance of narrow features for zero pump to probe detuning with 
a width determined by the effective ground state relaxation rate was predicted for open two-level systems [5]. In the present case this feature should be seen as the consequence of the population transfer, due to spontaneous emission, between pairs of levels connected by the fields. A detailed look into the evolution of this narrow resonance with the pump field power (for $\Delta=2 \Gamma$ ) is presented in Fig. 6. The resonance at $\delta=0$ is negative (dip) for $\Omega_{1}>0.4 \Gamma$. This result is to be compared with the contribution to the nonlinear atomic polarization arising from the different pairs of Zeeman sublevels with $m_{e}=m_{g}$. These contributions can be extracted from the calculation after identification of the density matrix coefficients corresponding to a given value of $\left|m_{i}\right|$ $(i=e, g)$. Figs. 7 and 8 represent the square modulus of the nonlinear atomic polarization contributions arising from the Zeeman sublevels pairs with $\left|m_{g}\right|=0$ and $\left|m_{g}\right|=1$ respectively. Only in the case of $\left|m_{g}\right|=0$ and for the largest value of $\Omega_{1}$ the central resonance is opposite to the lateral sidebands. In all other cases this resonance has the same sign than the lateral sidebands. This is always the case for $\left|m_{g}\right|=1$. Consequently, the dip observed at $\delta=0$ in the NDFWM spectra in Fig. 6 is the result of (destructive) quantum interference between the two contributions coming from $\left|m_{g}\right|=0,1$. Indeed, the two contributions have opposite phase over all the considered range of $\Omega_{1}$.

The NDFWM spectra obtained for perpendicular linear pump and probe polarizations (Fig. 9) are rather different to the ones presented above [14]. For all the considered pump field intensity range the spectra are dominated by the features occurring around $\delta=0$. In fact for $\Omega_{1} \lesssim 2 \Gamma$ the spectral sidebands are barely visible and the spectra is mainly composed by a large central resonance with a width of the order of $\gamma[8]$. This configuration produces for $\Omega_{1} \approx \Gamma$ the largest NDFWM yield [13]. For increasing $\Omega_{1}$ the central peak splits into two components. Also in this case the spectra are asymmetric for $\Delta \neq 0$. The main features of these spectra can be qualitatively understood using the dressed states picture of the degenerate atomic system in the presence of the pump field. A similar analysis was carried in [15](Appendix) to examine the probe absorption spectra of driven DTLS in this configuration. The dressed-state energy level scheme for a $F_{g}=1 \rightarrow F_{e}=2$ transition driven by a $\pi$ polarized pump is presented in Fig. 10 following the conventions adopted in [15]. NDFWM resonances are expected to occur when the probe field is resonant with a transitions between dressed levels that are coupled to the pump field photons. Four different probe frequencies satisfy this condition. The corresponding values of $\delta$ (see Table II in 15]) are indicated with solid arrows in the two lower spectra of Fig. 9. They correspond to the main features in the NDFWM spectra. From a simple dressed-state analysis, no resonance is expected to occur for the probe frequencies corresponding to transitions ending in the $|1 ", N\rangle$ dressed-atom levels since these states are not coupled to the pump photons due to the $m_{e}=m_{g}$ selection rule valid for a $\pi$ polarized pump. Nevertheless small features appear in the calculated spectra at these positions (dashed arrows in Fig. 9). They are due to non-secular terms usually neglected in the dressed atom approach.

\section{EXPERIMENTAL OBSERVATION OF NDFWM IN COLD CESIUM.}

The observation of the NDFWM spectra was performed in a sample of cold cesium atoms produced in a magneto-optical trap (MOT). Light from a Ti:sapphire laser, nearly resonant with the cesium cycling transition $6 S_{1 / 2}(F=4) \rightarrow 6 P_{3 / 2}(F=5)$, was employed both for the trapping of the atoms and for the investigation of the NDFWM. The experimental setup is shown schematically in Fig. 11. The frequency of the Ti:sapphire laser is red-detuned by approximately two natural linewidth $(\Gamma / 2 \pi=5.3 \mathrm{MHz})$. A repumping diode laser, not shown in the figure, recycles the population lost to the $6 S_{1 / 2}(F=3)$ ground state. We use a backwards FWM configuration, where the two counter-propagating pump fields, the forward (F) and backward (B), have the same frequency and the same linear polarization. The probe beam $(\mathrm{P})$, is linearly polarized and makes a small angle $\left(\theta=4^{\circ}\right)$ with the pumping beams. The probe beam has its frequency scanned around the frequency of the pump beams with the help of two acousto-optic modulators as shown in Fig. 11. The relative polarization between the pump and the probe fields is controlled by a half-wave plate. The generated (nearly) phase conjugated signal (PC), which propagates in opposite direction with respect to beam $\mathrm{P}$, is reflected out of a 50/50 beam splitter and detected by a fast photodiode. The trapping beams are switched on and off by a mechanical chopper with a transmission duty cycle of $95 \%$. The number of cold atoms was estimated by measuring the absorption of the probe beam and is of order of $10^{7}$. The NDFWM spectra were recorded within $\mathrm{a} \sim 1 \mathrm{~ms}$ time interval during which the trapping beams were blocked and the quadrupole magnetic field was turned off. Each of the F and B pump beams have an intensity of $7 \mathrm{~mW} / \mathrm{cm}^{2}$. The probe beam intensity is approximately equal to $0.7 \mathrm{~mW} / \mathrm{cm}^{2}$. No significant modification of the spectra was observed at lower probe beam intensities. The maximum NDFWM power generated was of the order of $1 \mu W$. Typical spectra, recorded as a function of the pump to probe frequency offset $\delta$, are shown in Figs. 12a) and 13a) for the probe beam polarization respectively parallel and perpendicular to the pump beam polarization. 


\section{DISCUSSION.}

The comparison of the observed spectra with the theoretical predictions presented above is not direct. While the calculation applies to a homogeneous ensemble of atoms at rest, the PC signal is the result of cooperative emission of atoms under different excitation conditions. In the experiment the pump field, at a given position in the sample, is due to the combined incidence of the $\mathrm{F}$ and $\mathrm{B}$ beams. The counterpropagating geometry of these beams produces a standing wave, consequently, the pump field intensity is spatially modulated. In general the pump field polarization may be spatial dependent. However, in the present study we have restricted ourselves to the case where the beams F and B have the same linear polarization and consequently no spatial variation of the polarization occurs. Also, the PC signal is sensitive to propagation effects in the atomic sample which are responsible for the phase matching condition and for possible spatial dependent amplification or depletion of the NDFWM through the medium. Finally, one should remind that the phase matching condition imposes constraints on the polarization components that may be present in the PC beam (only transverse components). However, in the two polarization cases considered in the experiment, linear parallel and linear perpendicular pump and probe polarizations, due to symmetry, the nonlinear atomic polarization should be parallel to the probe polarization (in the absence of magnetic field). In consequence, all polarization components of the NDFWM field are able to propagate along the PC beam.

In order to allow the comparison between the theoretical prediction and the experimental observation, we have incorporated into the calculation the intensity distribution of the pump field in the atomic sample. Propagation effects were not considered. Such approach, expected to be valid for an optically thin sample, is only approximative in our case since the peak probe absorption is around $50 \%$. Assuming that the $\mathrm{F}$ and $\mathrm{B}$ beams produce a perfect standing wave, we have considered a sine wave distribution of $\Omega_{1}$ in the interval $0 \leq \Omega_{1} \leq \Omega_{1 M A X}$. For each value of $\Omega_{1}$, the NDFWM field was calculated assuming that a steady state is reached. The total NDFWM field was taken as the sum of the contributions for each $\Omega_{1}$. Finally the NDFWM power correspond to the square modulus of the total field. The calculations were performed for an $F_{g}=4 \rightarrow F_{e}=5$ transition with $\Delta=-2 \Gamma$ (corresponding to the experimental conditions) and $\gamma=0.01 \Gamma$. No magnetic field was considered. The value of $\Omega_{1 M A X}$ was adjusted to fit the experimental spectra. The best agreement was obtained for $\Omega_{1 M A X}=18 \Gamma$.

Figure 12 $\mathrm{b}$ ) represents the calculated spectrum for linear and parallel pump and probe polarizations with the parameters corresponding to the experimental conditions. The spectrum is dominated by the two sidebands.
The width of the sidebands is mainly due to the inhomogeneity of the Rabi frequency. The largest values of $\Omega_{1}$ are responsible for the central peak presenting a width of the order of $\Gamma$ while the atoms corresponding to $\Omega_{1} \lesssim \Gamma$ are responsible for the narrow dip present at $\delta=0$. The main features of the experimental spectrum are well reproduced. Some differences appear in the amplitude and shape of the narrow resonance around $\delta=0$. According to the theoretical considerations presented above, this narrow resonance is the consequence of the coupling between different Zeeman sublevels pairs through spontaneous emission. Its width is governed by the time of flight relaxation rate $\gamma$ which effectively plays the role of a ground-state decay rate. For cold atoms, the average time of flight across the exciting beams is rather long (more than $1 \mathrm{~ms}$ for a $1 \mathrm{~mm}$ diameter beam) and the corresponding value of $\gamma<3 \times 10^{-5} \Gamma$ too small to account for the observed resonance. However, for typical MOT temperatures, the average time os flight across one spatial period of the stationary wave produced by the $\mathrm{F}$ and $\mathrm{B}$ beams is three orders of magnitude shorter and correspond to $\gamma \sim 10^{-2} \Gamma$ as assumed in the calculation. At this point one should notice that a short travelling time across the standing wave pattern of the pump field in not compatible with our assumption of a steady state reached for each value of $\Omega_{1}$. This suggests that a more sophisticated theoretical approach, incorporating the atomic motion, would be more appropriate for a precise description of the spectra. In addition to the finite interaction time, another mechanism that can affect the narrow resonance around $\delta=0$ is the possible leakeage out of the closed two-level transition. In our case, this may occur though non resonant excitation of other excited state hyperfine levels. However the corresponding rate can be estimated to be smaller than $10^{-3} \Gamma$. Also, calculations carried for open transitions give spectral shapes very different from those observed in Fig. 12 .

The comparison between observed and calculated spectra in the case of linear and perpendicular pump and probe polarizations are presented in Fig. 13. The main features of the spectra are well reproduced by the calculation. Notice the significant increase in the maximum NDFWM yield with respect to the previous case. The spectrum is in the present case dominated by the narrow resonance at $\delta=0$. The width of this resonance is also given at low pump intensities by $\gamma$. As already pointed in [8] and [13], this configuration provides the largest NDFWM yield. The difference in the spectral profile existing between Figs. 12 and 13 constitutes a clear demonstration of the essential role of the Zeeman degeneracy and optical polarizations in the NDFWM process. 


\section{CONCLUSIONS.}

The process of NDFWM in a closed atomic transitions with $F_{e}=F_{g}+1$ has been examined both theoretically and experimentally. Large differences in the spectra are observed for different choices of the exciting fields polarizations revealing the crucial role of the internal level structure on the non-linear process. The spectra present distinct features which are determined by the different relaxation rates and characteristic frequencies of the system $\left(\Gamma, \gamma, \Omega_{1}, \Delta\right)[6]$. NDFWM was observed in a cold sample of cesium atoms in a PC experiment for two different choices of the pump and probe polarizations. In spite of the difference existing between the experimental conditions and the assumptions of the theory a good agreement between calculated and observed spectra was obtained. Nevertheless, some features of the spectra, associated to the longest relaxation processes, indicate the need for a more detailed theoretical approach including the atomic motion and spatial field distribution.

\section{ACKNOWLEDGMENTS.}

The authors acknowledge fruitful discussions with J.R. Rios Leite. This work was supported by $\mathrm{CNPq}$ (PRONEX), CAPES and FINEP (Brazilian agencies) and by CSIC, CONYCIT and PEDECIBA (Uruguayan agencies).

[1] N. Bloembergen, Nonlinear Optics, Benjamin, New York (1965).

[2] Y.R. Shen, The principles of nonlinear optics, J. Wiley \& Sons, New York (1984).

[3] L.J. Rothberg and N. Bloembergen, Phys. Rev. A 30, 820 (1984). L.J. Rothberg and N. Bloembergen, Phys. Rev. A 30, 2327 (1984).

[4] R.W. Boyd, M.G. Raymer, P. Narum and D.J. Harter, Phys. Rev. A 24, 411 (1981).

[5] D.G. Steel and J.T. Remillard, Phys. Rev. A 36, 4330 (1987).

[6] P.R. Berman, D.G. Steel, G. Khitrova and J. Liu, Phys. Rev. A 38, 252 (1988).

[7] O.K. Andersen, D. Lenstra and S. Stolte, Phys. Rev. A 60, 1672 (1999).

[8] A. Lezama, S. Barreiro,. A. Lipsich and A.M. Akulshin, Phys. Rev. A 61, 013801 (2000).

[9] J.W.R. Tabosa, G. Chen, Z. Hu, R.B. Lee and H.J. Kimble, Phys. Rev. Lett. 66, 3245 (1991).

[10] D. Grison, B. Lounis, C. Salomon, J.Y. Courtois and G. Grynberg, Europhys. Lett. 15, 149 (1991).

[11] L. Hilico, P. Verkerk and G. Grynberg, C. R. Acad. Sci. Paris 315, 285 (1992).
[12] B. Lounis, P. Verkerk, C. Salomon, J.Y. Courtois and G. Grynberg, Nonlinear Optics 5, 459 (1993).

[13] A.M. Akulshin, S.Barreiro and A. Lezama, Quantum Electronics 30, 189 (2000); [Kvantovaya Elektronika 30, 189 (2000)].

[14] J.F. Lam, D.G. Steel and R.A. McFarlane, Phys. Rev. Lett. 56, 1679 (1986).

[15] A. Lipsich, S. Barreiro, A.M. Akulshin and A. Lezama, Phys. Rev. A 61, 053803 (2000).

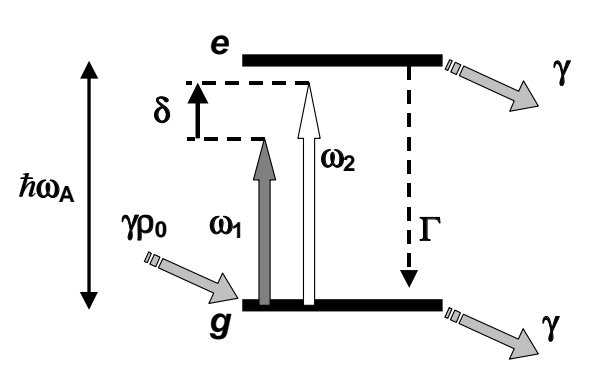

FIG. 1. Schematic representation of the configuration considered in the theory. $e$ and $g$ are degenerate levels. Solid vertical arrows represent the pump and probe fields (frequencies $\omega_{1}$ and $\omega_{2}$ respectively). Dashed arrow: decay by spontaneous emission. Oblique arrows: arrival and departure of the atoms into and from the interaction zone. The corresponding rates are indicated. 

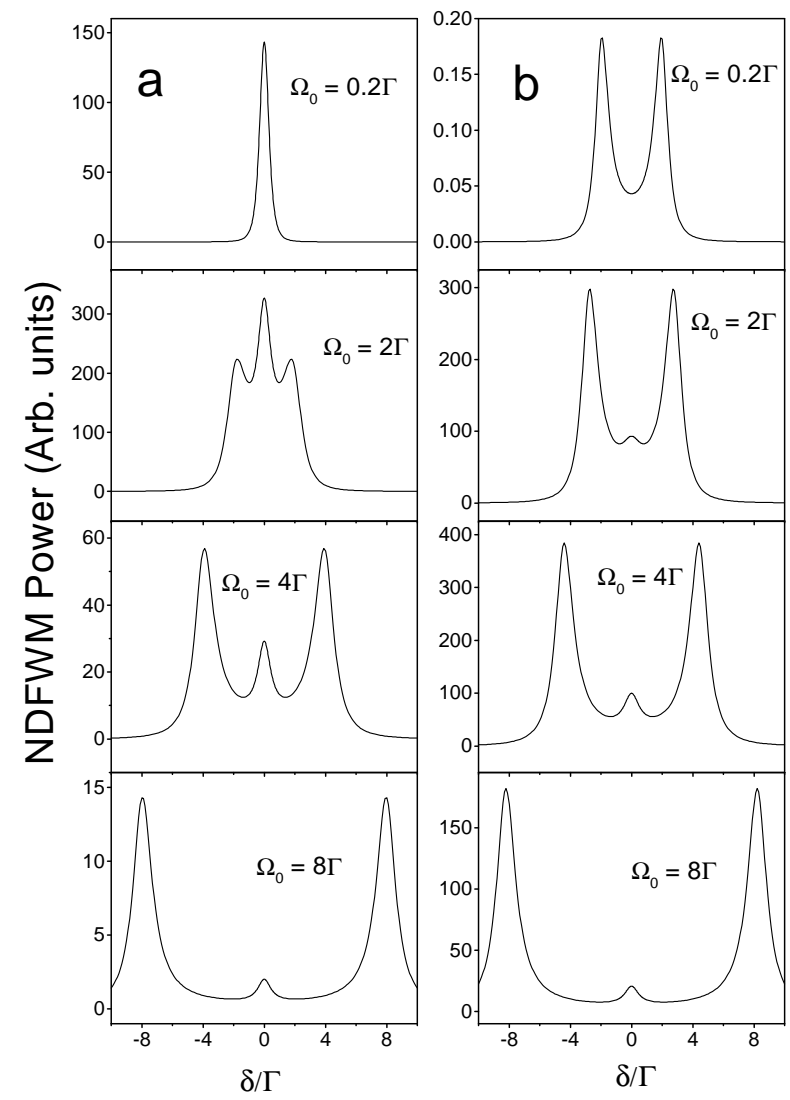

FIG. 2. Calculated NDFWM spectra for a pure two-level system. a) $\Delta=0$. b) $\Delta=2 \Gamma$.
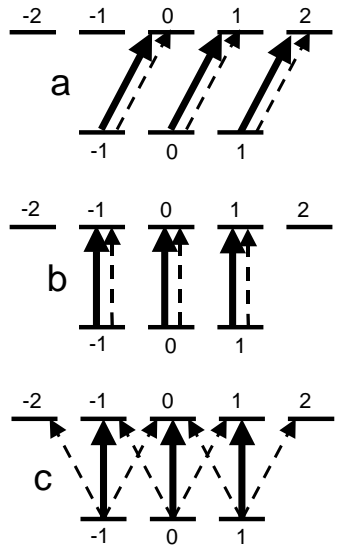

FIG. 3. Different level configurations corresponding to the transition $F_{g}=1 \rightarrow F_{e}=2$ for different choices of the pump and probe polarizations. a) Circular and equal. b) Linear parallel. c) Linear perpendicular. Solid (dashed) arrows correspond to the pump (probe) field. 

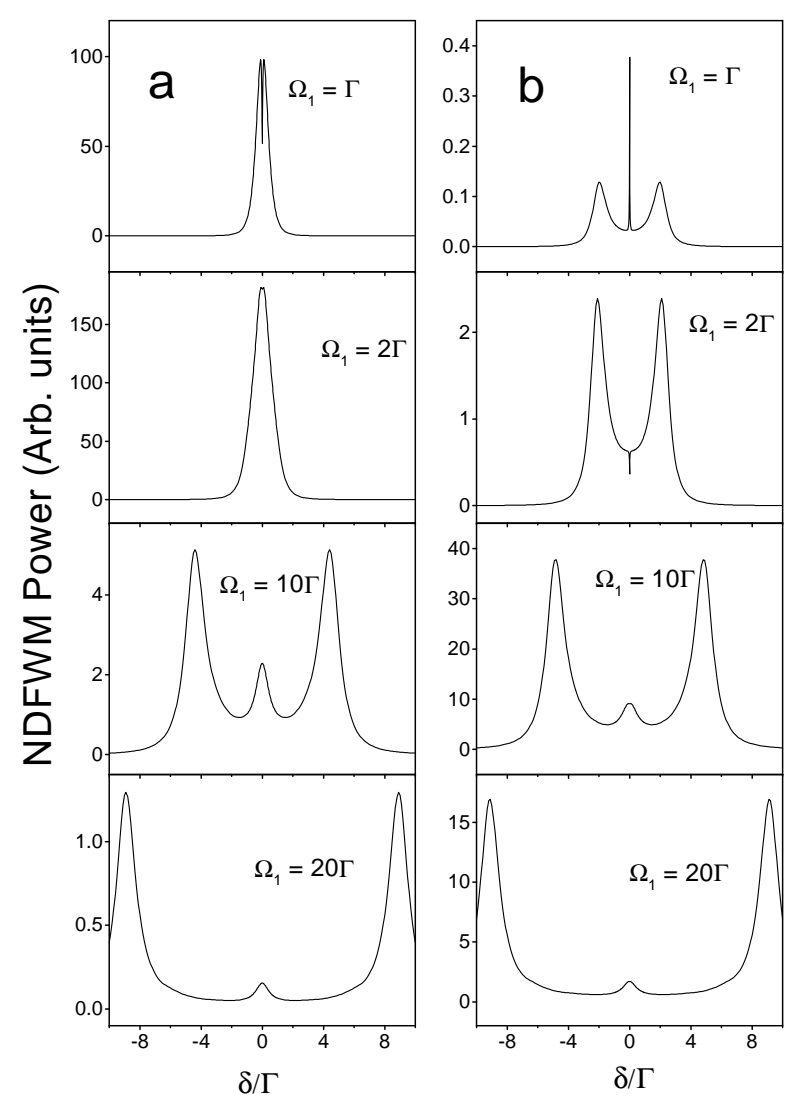

FIG. 4. Calculated NDFWM spectra for the transition $F_{g}=1 \rightarrow F_{e}=2$ for circular and equal pump and probe polarizations $(\gamma=0.01 \Gamma)$. a) $\Delta=0$. b) $\Delta=2 \Gamma$.
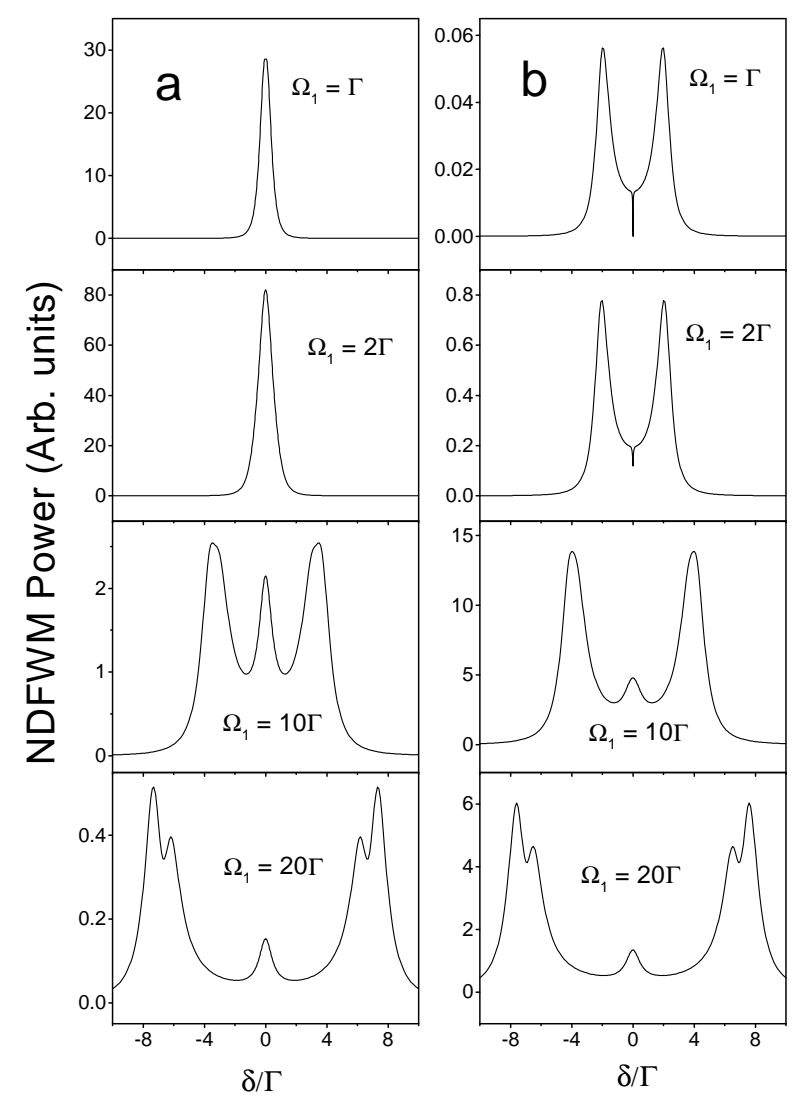

FIG. 5. Calculated NDFWM spectra for the transition $F_{g}=1 \rightarrow F_{e}=2$ for linear and parallel pump and probe polarizations $(\gamma=0.01 \Gamma)$. a) $\Delta=0$. b) $\Delta=2 \Gamma$.

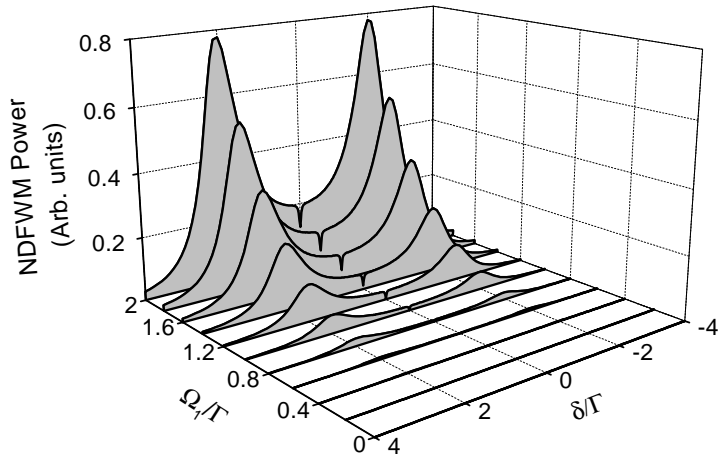

FIG. 6. NDFWM power spectra for the transition $F_{g}=1 \rightarrow F_{e}=2$ with $\Delta=2 \Gamma$ and $\gamma=0.01 \Gamma$ for different values of the pump field Rabi frequency $\Omega_{1}$. 


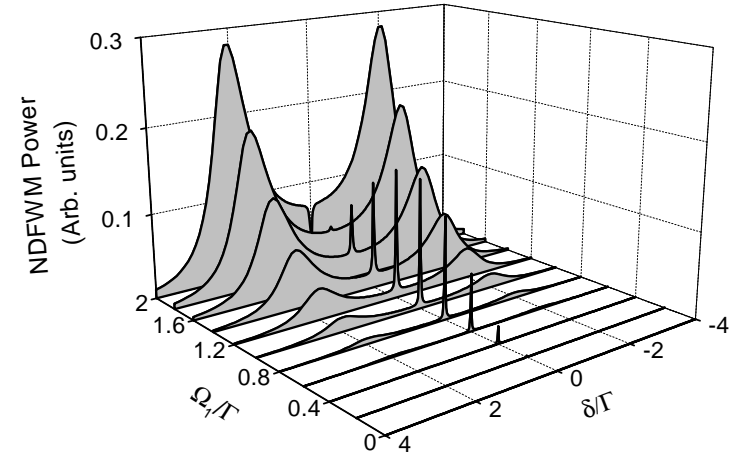

FIG. 7. Square modulus of the contribution to the atomic polarization at frequency $\omega_{3} \equiv \omega_{1}-\delta$ from the pair of Zeeman sublevels with $m=0$.

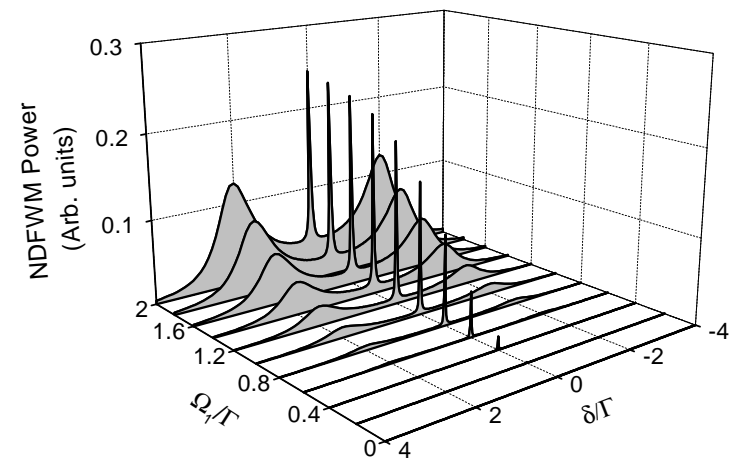

FIG. 8. Square modulus of the contribution to the atomic polarization at frequency $\omega_{3} \equiv \omega_{1}-\delta$ from the pairs of Zeeman sublevels with $|m|=1$.
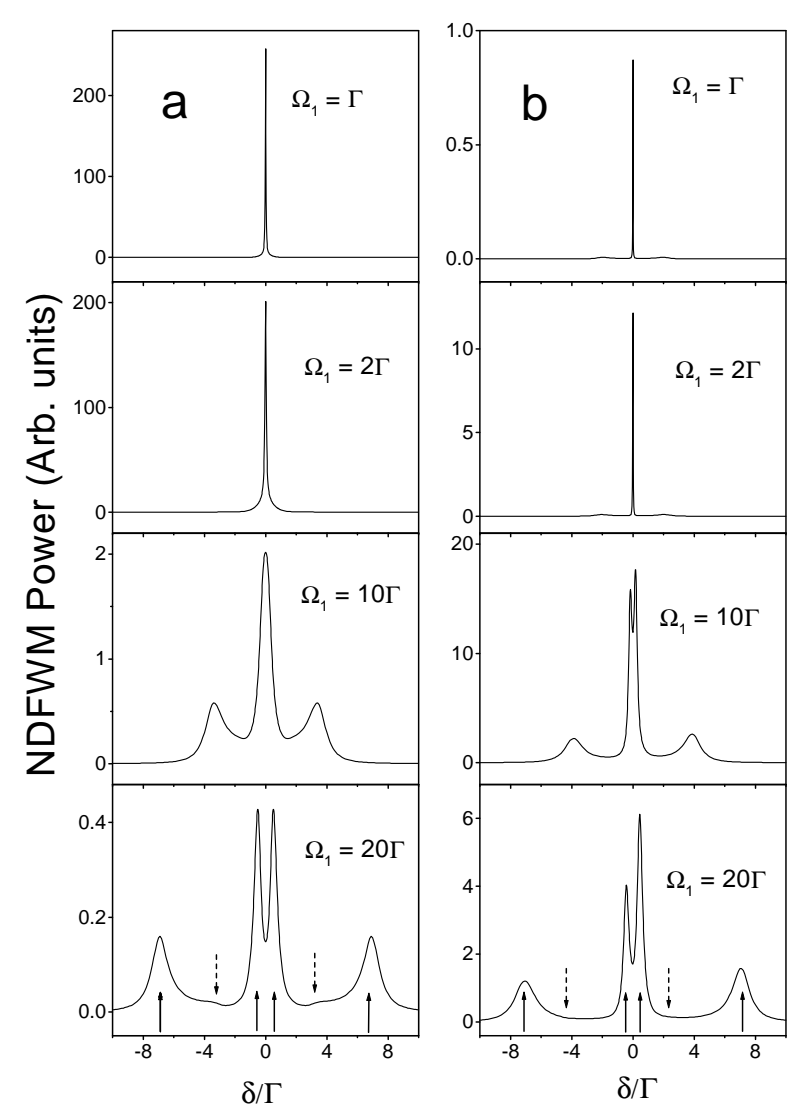

FIG. 9. Calculated NDFWM spectra for the transition $F_{g}=1 \rightarrow F_{e}=2$ for perpendicular linear pump and probe polarizations $(\gamma=0.01 \Gamma)$. a) $\Delta=0$. b) $\Delta=2 \Gamma$.

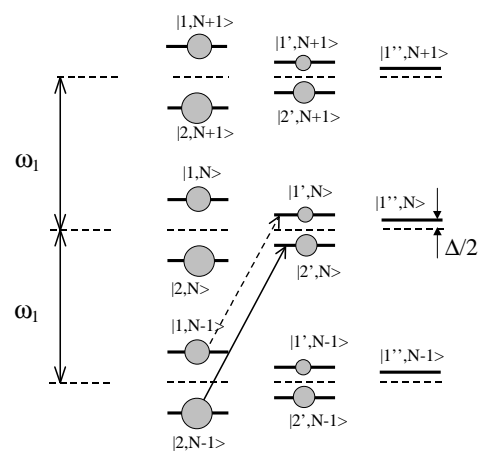


FIG. 10. Dressed atom energy level scheme for a transition $F_{g}=1 \rightarrow F_{e}=2$ driven by a $\pi$ polarized pump field. The notations are taken from Ref. [8]. The arrows illustrate two possible probe field transitions giving rise to resonances in the NDFWM spectra corresponding to opposite values of $\delta$.

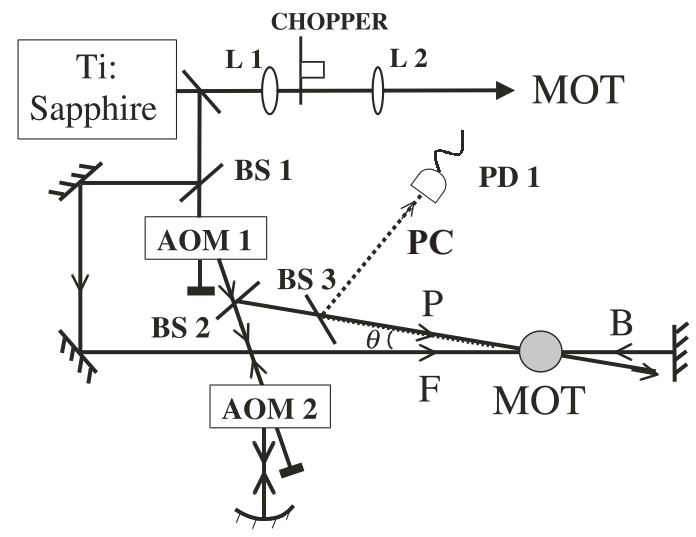

FIG. 11. a) Experimental setup. AOM: acousto-optic modulator. BS: beam splitter. PD: photodiode. MOT: magneto-optically trapped atomic sample.

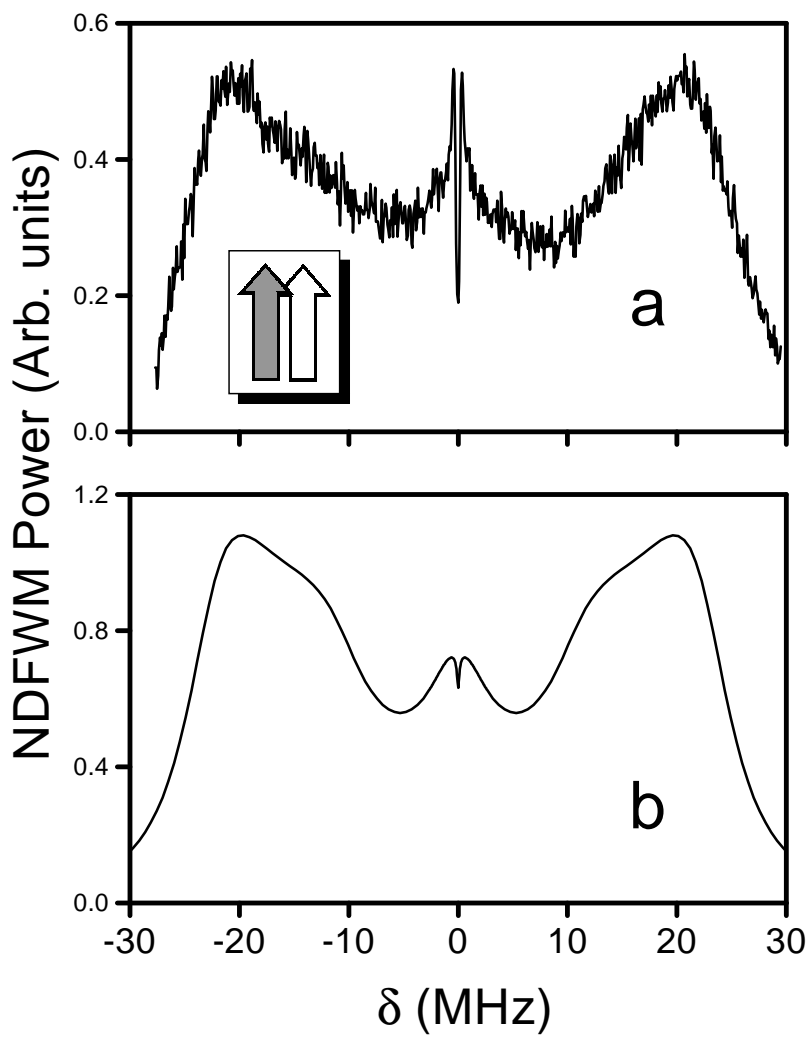

FIG. 12. Observed $a$ ) and calculated $b$ ) NDFWM spectra for linear and parallel pump and probe polarizations. The vertical axes scales are independent (the same scales are used in Fig.13). 


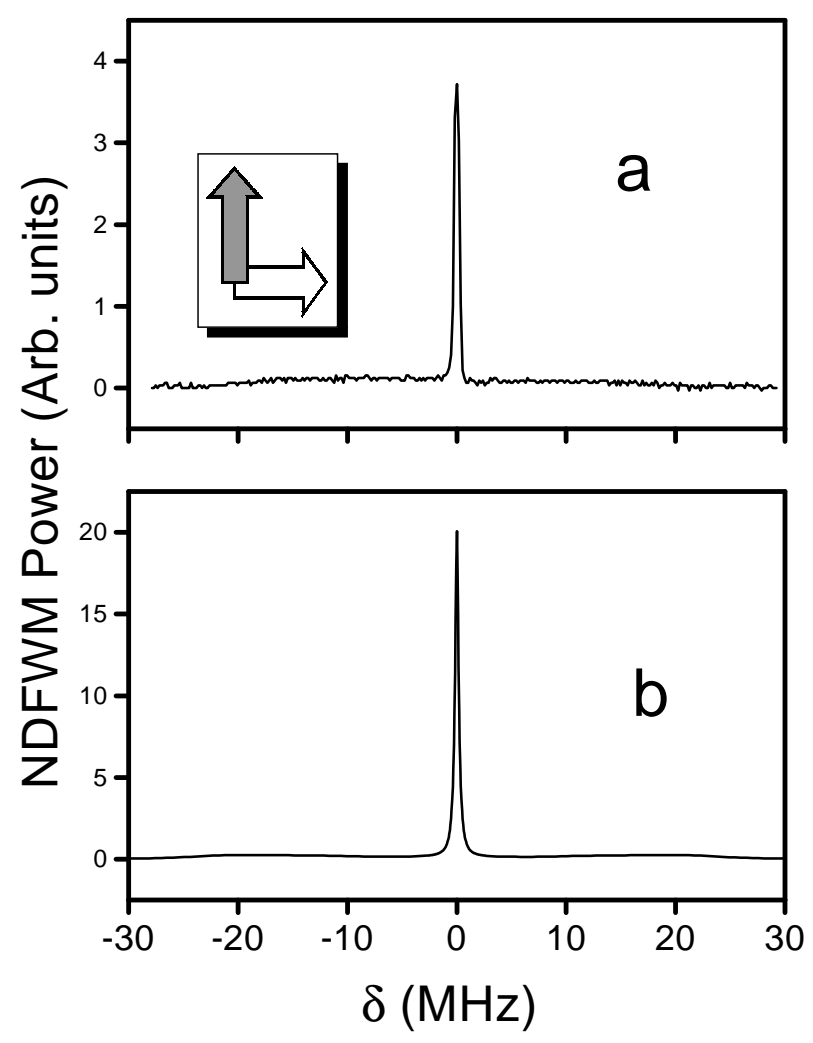

FIG. 13. Observed $a$ ) and calculated $b$ ) NDFWM spectra for linear and perpendicular pump and probe polarizations. The vertical axes scales are independent (the same scales are used in Fig.12. 